\title{
Exclusive electroproduction of two pions at HERA
}

\author{
The ZEUS Collaboration
}

H. Abramowicz ${ }^{45, \text { ah }}$, I. Abt ${ }^{35}$, L. Adamczyk ${ }^{13}$, M. Adamus ${ }^{54}$, R. Aggarwal $^{7, d}$, S. Antonelli ${ }^{4}$, P. Antonioli ${ }^{3}$, A. Antonov ${ }^{33}$, M. Arneodo ${ }^{50}$, D. Ashery ${ }^{45}$, V. Aushev ${ }^{26,27, z}$, Y. Aushev ${ }^{27, z, a a}$, O. Bachynska ${ }^{15}$, A. Bamberger ${ }^{19}$,

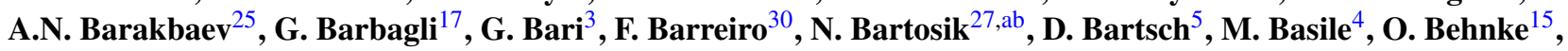
J. Behr ${ }^{15}$, U. Behrens ${ }^{15}$, L. Bellagamba ${ }^{3}$, A. Bertolin ${ }^{39}$, S. Bhadra ${ }^{57}$, M. Bindi ${ }^{4}$, C. Blohm ${ }^{15}$, V. Bokhonov ${ }^{26, z}$, T. Bołd ${ }^{13}$, K. Bondarenko ${ }^{27}$, E.G. Boos ${ }^{25}$, K. Borras ${ }^{15}$, D. Boscherini ${ }^{3}$, D. Bot ${ }^{15}$, I. Brock ${ }^{5}$, E. Brownson ${ }^{56}$, R. Brugnera ${ }^{40}$, N. Brümmer ${ }^{37}$, A. Bruni ${ }^{3}$, G. Bruni ${ }^{3}$, B. Brzozowska ${ }^{53}$, P.J. Bussey ${ }^{20}$, B. Bylsma ${ }^{37}$, A. Caldwell ${ }^{35}$, M. Capua $^{8}$, R. Carlin $^{40}$, C.D. Catterall ${ }^{57}$, S. Chekanov ${ }^{1}$, J. Chwastowski ${ }^{12, f}$, J. Ciborowski ${ }^{53, a l}$, R. Ciesielski ${ }^{15, h}$, L. Cifarelli ${ }^{4}$, F. Cindolo ${ }^{3}$, A. Contin ${ }^{4}$, A.M. Cooper-Sarkar ${ }^{38}$, N. Coppola ${ }^{15, i}$, M. Corradi ${ }^{3}$, F. Corriveau ${ }^{31}$, M. Costa ${ }^{49}$, G. D'Agostini ${ }^{43}$, F. Dal Corso ${ }^{39}$, J. del Peso ${ }^{30}$, R.K. Dementiev ${ }^{34}$, S. De Pasquale ${ }^{4, b}$, M. Derrick ${ }^{1}$, R.C.E. Devenish ${ }^{38}$, D. Dobur ${ }^{19, t}$, B.A. Dolgoshein ${ }^{33, \dagger}$, G. Dolinska ${ }^{26,27}$, A.T. Doyle ${ }^{20}$, V. Drugakov ${ }^{16}$, L.S. Durkin ${ }^{37}$, S. Dusini $^{39}$, Y. Eisenberg ${ }^{55}$, P.F. Ermolov ${ }^{34, \dagger}$, S. Eskreys ${ }^{12, \dagger}$, S. Fang ${ }^{15, j}$, S. Fazio ${ }^{8}$, J. Ferrando ${ }^{38}$, M.I. Ferrero ${ }^{49}$,

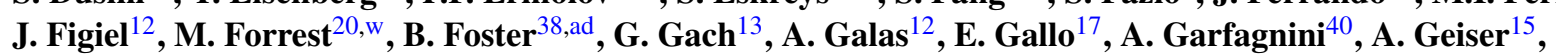

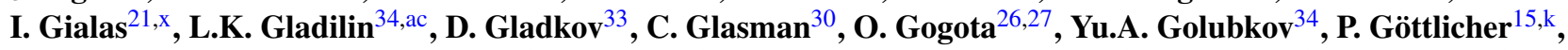
I. Grabowska-Bołd ${ }^{13}$, J. Grebenyuk ${ }^{15}$, I. Gregor ${ }^{15}$, G. Grigorescu ${ }^{36}$, G. Grzelak ${ }^{53}$, O. Gueta ${ }^{45}$, E. Gurvich ${ }^{45}$, M. Guzik ${ }^{13}$, C. Gwenlan ${ }^{38, \text { ae }}$, T. Haas ${ }^{15}$, W. Hain ${ }^{15}$, R. Hamatsu ${ }^{48}$, J.C. Hart ${ }^{44}$, H. Hartmann ${ }^{5}$, G. Hartner ${ }^{57}$,

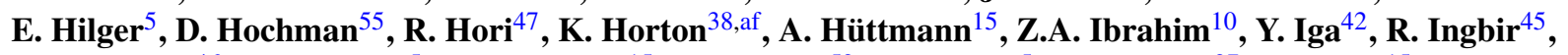
M. Ishitsuka ${ }^{46}$, H.-P. Jakob ${ }^{5}$, F. Januschek ${ }^{15}$, T.W. Jones ${ }^{52}$, M. Jüngst ${ }^{5}$, I. Kadenko ${ }^{27}$, B. Kahle ${ }^{15}$, S. Kananov ${ }^{45}$,

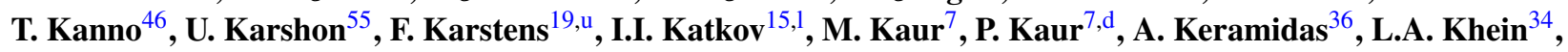
J.Y. Kim ${ }^{9}$, D. Kisielewska ${ }^{13}$, S. Kitamura ${ }^{48, a j}$, R. Klanner ${ }^{22}$, U. Klein ${ }^{15, m}$, E. Koffeman ${ }^{36}$, P. Kooijman ${ }^{36}$,

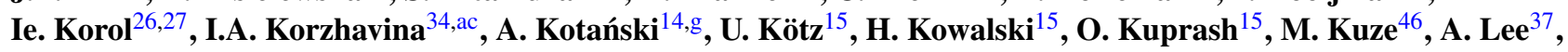
B.B. Levchenko ${ }^{34}$, A. Levy ${ }^{45, a}$, V. Libov ${ }^{15}$, S. Limentani ${ }^{40}$, T.Y. Ling ${ }^{37}$, M. Lisovyi ${ }^{15}$, E. Lobodzinska ${ }^{15}$, W. Lohmann ${ }^{16}$, B. Löhr ${ }^{15}$, E. Lohrmann ${ }^{22}$, K.R. Long ${ }^{23}$, A. Longhin ${ }^{39}$, D. Lontkovskyi ${ }^{15}$, O.Yu. Lukina ${ }^{34}$,

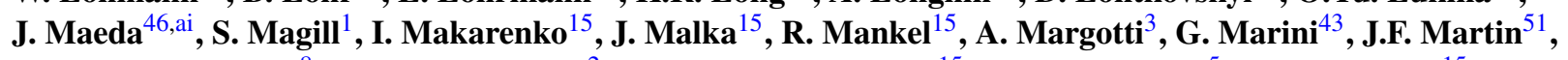
A. Mastroberardino ${ }^{8}$, M.C.K. Mattingly ${ }^{2}$, I.-A. Melzer-Pellmann ${ }^{15}$, S. Mergelmeyer ${ }^{5}$, S. Miglioranzi ${ }^{15, n}$, F. Mohamad Idris ${ }^{10}$, V. Monaco ${ }^{49}$, A. Montanari ${ }^{15}$, J.D. Morris ${ }^{6, c}$, K. Mujkic $^{15, \mathrm{o}}$, B. Musgrave ${ }^{1}$, K. Nagano ${ }^{24}$,

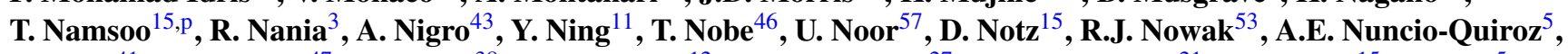
B.Y. Oh ${ }^{41}$, N. Okazaki ${ }^{47}$, K. Oliver ${ }^{38}$, K. Olkiewicz ${ }^{12}$, Yu. Onishchuk ${ }^{27}$, K. Papageorgiu ${ }^{21}$, A. Parenti ${ }^{15}$, E. Paul ${ }^{5}$, J.M. Pawlak ${ }^{53}$, B. Pawlik ${ }^{12}$, P.G. Pelfer ${ }^{18}$, A. Pellegrino ${ }^{36}$, W. Perlański ${ }^{53, a m}$, H. Perrey ${ }^{15}$, K. Piotrzkowski ${ }^{29}$, P. Pluciński $^{54, \text { an }}$, N.S. Pokrovskiy ${ }^{25}$, A. Polini ${ }^{3}$, A.S. Proskuryakov ${ }^{34}$, M. Przybycień ${ }^{13}$, A. Raval ${ }^{15}$, D.D. Reeder $^{56}$,

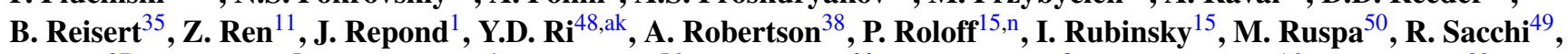
A. Salii $^{27}$, U. Samson ${ }^{5}$, G. Sartorelli ${ }^{4}$, A.A. Savin $^{56}$, D.H. Saxon ${ }^{20}$, M. Schioppa ${ }^{8}$, S. Schlenstedt ${ }^{16}$, P. Schleper ${ }^{22}$, W.B. Schmidke ${ }^{35}$, U. Schneekloth ${ }^{15}$, V. Schönberg ${ }^{5}$, T. Schörner-Sadenius ${ }^{15}$, J. Schwartz ${ }^{31}$, F. Sciulli ${ }^{11}$, L.M. Shcheglova ${ }^{34}$, R. Shehzadi ${ }^{5}$, S. Shimizu ${ }^{47, \mathrm{n}}$, I. Singh ${ }^{7, \mathrm{~d}}$, I.O. Skillicorn ${ }^{20}$, W. Słomiński ${ }^{14}$, W.H. Smith ${ }^{56}$, V. Sola ${ }^{49}$, A. Solano ${ }^{49}$, D. Son ${ }^{28}$, V. Sosnovtsev ${ }^{33}$, A. Spiridonov ${ }^{15, q}$, H. Stadie ${ }^{22}$, L. Stanco ${ }^{39}$, A. Stern ${ }^{45}$, T.P. Stewart ${ }^{51}$, A. Stifutkin ${ }^{33}$, P. Stopa ${ }^{12}$, S. Suchkov ${ }^{33}$, G. Susinno ${ }^{8}$, L. Suszycki ${ }^{13}$, J. Sztuk-Dambietz ${ }^{22}$, D. Szuba ${ }^{22}$,

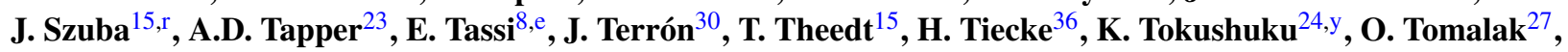

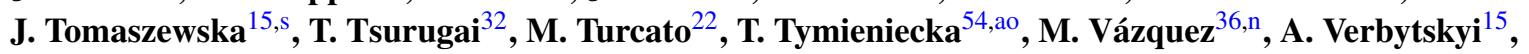
O. Viazlo $^{26,27}$, N.N. Vlasov ${ }^{19, v}$, O. Volynets $^{27}$, R. Walczak ${ }^{38}$, W.A.T. Wan Abdullah ${ }^{10}$, J.J. Whitmore ${ }^{41, a g}$, L. Wiggers ${ }^{36}$, M. Wing ${ }^{52}$, M. Wlasenko ${ }^{5}$, G. Wolf ${ }^{15}$, H. Wolfe ${ }^{56}$, K. Wrona ${ }^{15}$, A.G. Yagües-Molina ${ }^{15}$, S. Yamada $^{24}$, Y. Yamazaki $^{24, \mathrm{z}}$, R. Yoshida ${ }^{1}$, C. Youngman ${ }^{15}$, A.F. Żarnecki ${ }^{53}$, L. Zawiejski ${ }^{12}$, O. Zenaiev $^{15}$, W. Zeuner ${ }^{15, \mathrm{n}}$, B.O. Zhautykov ${ }^{25}$, N. Zhmak ${ }^{26, z}$, C. Zhou ${ }^{31}$, A. Zichichi ${ }^{4}$, Z. Zolkapli ${ }^{10}$, M. Zolko ${ }^{27}$, D.S. Zotkin ${ }^{34}$

\footnotetext{
${ }^{1}$ Argonne National Laboratory, Argonne, IL 60439-4815, USA ${ }^{\text {A }}$

${ }^{2}$ Andrews University, Berrien Springs, MI 49104-0380, USA

${ }^{3}$ INFN Bologna, Bologna, Italy ${ }^{B}$
} 
${ }^{4}$ University and INFN Bologna, Bologna, Italy ${ }^{\mathrm{B}}$

${ }^{5}$ Physikalisches Institut der Universität Bonn, Bonn, Germany ${ }^{C}$

${ }^{6}$ H.H. Wills Physics Laboratory, University of Bristol, Bristol, $\mathrm{UK}^{\mathrm{D}}$

${ }^{7}$ Panjab University, Department of Physics, Chandigarh, India

${ }^{8}$ Calabria University, Physics Department and INFN, Cosenza, Italy ${ }^{\mathrm{B}}$

${ }^{9}$ Institute for Universe and Elementary Particles, Chonnam National University, Kwangju, South Korea

${ }^{10}$ Jabatan Fizik, Universiti Malaya, 50603 Kuala Lumpur, Malaysia ${ }^{\mathrm{E}}$

${ }^{11}$ Nevis Laboratories, Columbia University, Irvington on Hudson, NY 10027, USA ${ }^{\mathrm{F}}$

${ }^{12}$ The Henryk Niewodniczanski Institute of Nuclear Physics, Polish Academy of Sciences, Krakow, Poland ${ }^{G}$

${ }^{13}$ AGH-University of Science and Technology, Faculty of Physics and Applied Computer Science, Krakow, Poland ${ }^{\mathrm{H}}$

${ }^{14}$ Department of Physics, Jagellonian University, Cracow, Poland

${ }^{15}$ Deutsches Elektronen-Synchrotron DESY, Hamburg, Germany

${ }^{16}$ Deutsches Elektronen-Synchrotron DESY, Zeuthen, Germany

${ }^{17}$ INFN Florence, Florence, Italy ${ }^{\mathrm{B}}$

${ }^{18}$ University and INFN Florence, Florence, Italy ${ }^{\mathrm{B}}$

${ }^{19}$ Fakultät für Physik der Universität Freiburg i.Br., Freiburg i.Br., Germany

${ }^{20}$ School of Physics and Astronomy, University of Glasgow, Glasgow, UK ${ }^{\mathrm{D}}$

${ }^{21}$ Department of Engineering in Management and Finance, Univ. of the Aegean, Chios, Greece

${ }^{22}$ Hamburg University, Institute of Experimental Physics, Hamburg, Germany ${ }^{\mathrm{I}}$

${ }^{23}$ Imperial College London, High Energy Nuclear Physics Group, London, $\mathrm{UK}^{\mathrm{D}}$

${ }^{24}$ Institute of Particle and Nuclear Studies, KEK, Tsukuba, Japan ${ }^{\mathrm{J}}$

${ }^{25}$ Institute of Physics and Technology of Ministry of Education and Science of Kazakhstan, Almaty, Kazakhstan

${ }^{26}$ Institute for Nuclear Research, National Academy of Sciences, Kyiv, Ukraine

${ }^{27}$ Department of Nuclear Physics, National Taras Shevchenko University of Kyiv, Kyiv, Ukraine

${ }^{28}$ Kyungpook National University, Center for High Energy Physics, Daegu, South Korea ${ }^{\mathrm{K}}$

${ }^{29}$ Institut de Physique Nucléaire, Université Catholique de Louvain, Louvain-la-Neuve, Belgium ${ }^{\mathrm{L}}$

${ }^{30}$ Departamento de Física Teórica, Universidad Autónoma de Madrid, Madrid, Spain ${ }^{\mathrm{M}}$

${ }^{31}$ Department of Physics, McGill University, Montréal, Québec, Canada H3A 2T8 ${ }^{\mathrm{N}}$

${ }^{32}$ Meiji Gakuin University, Faculty of General Education, Yokohama, Japan ${ }^{\mathrm{J}}$

${ }^{33}$ Moscow Engineering Physics Institute, Moscow, Russia ${ }^{\mathrm{O}}$

${ }^{34}$ Moscow State University, Institute of Nuclear Physics, Moscow, Russia ${ }^{\mathrm{P}}$

${ }^{35}$ Max-Planck-Institut für Physik, München, Germany

${ }^{36}$ NIKHEF and University of Amsterdam, Amsterdam, Netherlands $Q$

${ }^{37}$ Physics Department, Ohio State University, Columbus, OH 43210, USA ${ }^{\mathrm{A}}$

${ }^{38}$ Department of Physics, University of Oxford, Oxford, UK ${ }^{\mathrm{D}}$

${ }^{39}$ INFN Padova, Padova, Italy ${ }^{\mathrm{B}}$

${ }^{40}$ Dipartimento di Fisica dell' Università and INFN, Padova, Italy ${ }^{\mathrm{B}}$

${ }^{41}$ Department of Physics, Pennsylvania State University, University Park, PA 16802, USA ${ }^{\mathrm{F}}$

${ }^{42}$ Polytechnic University, Sagamihara, Japan ${ }^{\mathrm{J}}$

${ }^{43}$ Dipartimento di Fisica, Università 'La Sapienza' and INFN, Rome, Italy ${ }^{B}$

${ }^{44}$ Rutherford Appleton Laboratory, Chilton, Didcot, Oxon, UK ${ }^{\mathrm{D}}$

${ }^{45}$ Raymond and Beverly Sackler Faculty of Exact Sciences, School of Physics, Tel Aviv University, Tel Aviv, Israel ${ }^{\mathrm{R}}$

${ }^{46}$ Department of Physics, Tokyo Institute of Technology, Tokyo, Japan ${ }^{\mathrm{J}}$

${ }^{47}$ Department of Physics, University of Tokyo, Tokyo, Japan ${ }^{\mathrm{J}}$

${ }^{48}$ Tokyo Metropolitan University, Department of Physics, Tokyo, Japan ${ }^{\mathrm{J}}$

${ }^{49}$ Università di Torino and INFN, Torino, Italy ${ }^{\mathrm{B}}$

${ }^{50}$ Università del Piemonte Orientale, Novara, and INFN, Torino, Italy ${ }^{\mathrm{B}}$

${ }_{51}^{51}$ Department of Physics, University of Toronto, Toronto, Ontario, Canada M5S $1 \mathrm{~A} 7^{\mathrm{N}}$

${ }^{52}$ Physics and Astronomy Department, University College London, London, $\mathrm{UK}^{\mathrm{D}}$

${ }^{53}$ Faculty of Physics, University of Warsaw, Warsaw, Poland

${ }^{54}$ National Centre for Nuclear Research, Warsaw, Poland

${ }^{55}$ Department of Particle Physics and Astrophysics, Weizmann Institute, Rehovot, Israel

${ }^{56}$ Department of Physics, University of Wisconsin, Madison, WI 53706, USA ${ }^{\mathrm{A}}$

${ }^{57}$ Department of Physics, York University, Toronto, Ontario, Canada M3J 1P3 ${ }^{\mathrm{N}}$

Received: 21 November 2011 / Published online: 25 January 2012

(C) The Author(s) 2012. This article is published with open access at Springerlink.com

Abstract The exclusive electroproduction of two pions in the mass range $0.4<M_{\pi \pi}<2.5 \mathrm{GeV}$ has been studied with the ZEUS detector at HERA using an integrated luminosity of $82 \mathrm{pb}^{-1}$. The analysis was carried out in the kine- matic range of $2<Q^{2}<80 \mathrm{GeV}^{2}, 32<W<180 \mathrm{GeV}$ and $|t|<0.6 \mathrm{GeV}^{2}$, where $Q^{2}$ is the photon virtuality, $W$ is the photon-proton centre-of-mass energy and $t$ is the squared four-momentum transfer at the proton vertex. The 
two-pion invariant-mass distribution is interpreted in terms of the pion electromagnetic form factor, $\left|F\left(M_{\pi \pi}\right)\right|$, assuming that the studied mass range includes the contributions of the $\rho, \rho^{\prime}$ and $\rho^{\prime \prime}$ vector-meson states. The masses and widths of the resonances were obtained and the $Q^{2}$ dependence of the cross-section ratios $\sigma\left(\rho^{\prime} \rightarrow \pi \pi\right) / \sigma(\rho)$ and $\sigma\left(\rho^{\prime \prime} \rightarrow \pi \pi\right) / \sigma(\rho)$ was extracted. The pion form factor obtained in the present analysis is compared to that obtained in $e^{+} e^{-} \rightarrow \pi^{+} \pi^{-}$.

a e-mail: levy@alzt.tau.ac.il

${ }^{\text {A }}$ Supported by the US Department of Energy

${ }^{\mathrm{B}}$ Supported by the Italian National Institute for Nuclear Physics (INFN)

${ }^{\mathrm{C}}$ Supported by the German Federal Ministry for Education and Research (BMBF), under contract No. 05 H09PDF

${ }^{D}$ Supported by the Science and Technology Facilities Council, UK

${ }^{\mathrm{E}}$ Supported by an FRGS grant from the Malaysian government

${ }^{F}$ Supported by the US National Science Foundation. Any opinion, findings and conclusions or recommendations expressed in this material are those of the authors and do not necessarily reflect the views of the National Science Foundation

${ }^{\mathrm{G}}$ Supported by the Polish Ministry of Science and Higher Education as a scientific project No. DPN/N188/DESY/2009

${ }^{\mathrm{H}}$ Supported by the Polish Ministry of Science and Higher Education and its grants for Scientific Research

${ }^{\text {IS }}$ upported by the German Federal Ministry for Education and Research (BMBF), under contract No. 05h09GUF, and the SFB 676 of the Deutsche Forschungsgemeinschaft (DFG)

${ }^{\mathrm{J}}$ Supported by the Japanese Ministry of Education, Culture, Sports, Science and Technology (MEXT) and its grants for Scientific Research

${ }^{\mathrm{K}}$ Supported by the Korean Ministry of Education and Korea Science and Engineering Foundation

${ }^{\mathrm{L}}$ Supported by FNRS and its associated funds (IISN and FRIA) and by an Inter-University Attraction Poles Programme subsidised by the Belgian Federal Science Policy Office

${ }^{\mathrm{M}}$ Supported by the Spanish Ministry of Education and Science through funds provided by CICYT

${ }^{\mathrm{N}}$ Supported by the Natural Sciences and Engineering Research Council of Canada (NSERC)

OPartially supported by the German Federal Ministry for Education and Research (BMBF)

${ }^{\text {P}}$ Supported by RF Presidential grant N 4142.2010.2 for Leading Scientific Schools, by the Russian Ministry of Education and Science through its grant for Scientific Research on High Energy Physics and under contract No.02.740.11.0244

QSupported by the Netherlands Foundation for Research on Matter (FOM)

${ }^{\mathrm{R}}$ Supported by the Israel Science Foundation

${ }^{\mathrm{b}}$ Now at University of Salerno, Italy

${ }^{\mathrm{c}}$ Now at Queen Mary University of London, United Kingdom

${ }^{\mathrm{d}}$ Also funded by Max Planck Institute for Physics, Munich, Germany

${ }^{e}$ Also Senior Alexander von Humboldt Research Fellow at Hamburg University, Institute of Experimental Physics, Hamburg, Germany

\section{Introduction}

Exclusive electroproduction of vector mesons takes place through a virtual photon $\gamma^{*}$ by means of the process $\gamma^{*} p \rightarrow V p$. At large values of the centre-of-mass energy, $W$, this is usually viewed as a three-step process; the virtual photon $\gamma^{*}$ fluctuates into a $q \bar{q}$ pair which interacts with the proton through a two-gluon ladder and hadronizes into a vector meson, $V$. The production of ground-state vector mesons, $V=\rho, \omega, \phi, J / \psi, \Upsilon$, which are $1 S$ triplet

${ }^{f}$ Also at Cracow University of Technology, Faculty of Physics, Mathemathics and Applied Computer Science, Poland

${ }^{\mathrm{g}}$ Supported by the research grant No. 1 P03B 04529 (2005-2008)

${ }^{\mathrm{h}}$ Now at Rockefeller University, New York, NY 10065, USA

${ }^{\mathrm{i}}$ Now at DESY group FS-CFEL-1

${ }^{\mathrm{j}}$ Now at Institute of High Energy Physics, Beijing, China

${ }^{\mathrm{k}}$ Now at DESY group FEB, Hamburg, Germany

${ }^{1}$ Also at Moscow State University, Russia

${ }^{\mathrm{m}}$ Now at University of Liverpool, UK

${ }^{\mathrm{n}}$ Now at CERN, Geneva, Switzerland

${ }^{\circ}$ Also affiliated with University College London, UK

pNow at Goldman Sachs, London, UK

qAlso at Institute of Theoretical and Experimental Physics, Moscow, Russia

${ }^{\mathrm{r}}$ Also at FPACS, AGH-UST, Cracow, Poland

${ }^{\text {s} P a r t i a l l y ~ s u p p o r t e d ~ b y ~ W a r s a w ~ U n i v e r s i t y, ~ P o l a n d ~}$

${ }^{t}$ Now at Istituto Nucleare di Fisica Nazionale (INFN), Pisa, Italy

${ }^{u}$ Now at Haase Energie Technik AG, Neumünster, Germany

${ }^{\mathrm{v}}$ Now at Department of Physics, University of Bonn, Germany

${ }^{\mathrm{w}}$ Now at Biodiversität und Klimaforschungszentrum (BiK-F), Frankfurt, Germany

${ }^{x}$ Also affiliated with DESY, Germany

${ }^{\mathrm{y}}$ Also at University of Tokyo, Japan

${ }^{\mathrm{z}}$ Now at Kobe University, Japan

${ }^{\mathrm{z}}$ Supported by DESY, Germany

${ }^{\dagger}$ Deceased

${ }^{\text {aa }}$ Member of National Technical University of Ukraine, Kyiv Polytechnic Institute, Kyiv, Ukraine

${ }^{a b}$ Member of National University of Kyiv-Mohyla Academy, Kyiv, Ukraine

${ }^{\text {ac }}$ Partly supported by the Russian Foundation for Basic Research, grant 11-02-91345-DFG_a

${ }^{\text {ad }}$ Alexander von Humboldt Professor; also at DESY and University of Oxford

${ }^{\text {ae }}$ STFC Advanced Fellow

${ }^{\text {af Nee Korcsak-Gorzo }}$

${ }^{\mathrm{ag}}$ This material was based on work supported by the National Science Foundation, while working at the Foundation

${ }^{\text {ah }}$ Also at Max Planck Institute for Physics, Munich, Germany, External Scientific Member

ai Now at Tokyo Metropolitan University, Japan

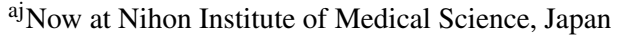

${ }^{\mathrm{ak}}$ Now at Osaka University, Osaka, Japan 
$q \bar{q}$ states, has been extensively studied at HERA, particularly in several recent publications [1-10]. As the virtuality, $Q^{2}$, of the photon increases, the process becomes hard and can be calculated in perturbative Quantum Chromodynamics (pQCD). Furthermore, by varying $Q^{2}$, and thus the size of the $q \bar{q}$ pair, sensitivity to the vector-meson wavefunction can be obtained by scanning it at different $q \bar{q}$ distance scales. Expectations in the QCD framework vary from calculations based only on the mass properties and typical size of the $q \bar{q}$ inside the vector-meson [11, 12], to those based on the details of the vector meson wave-function dependence on the size of the $q \bar{q}$ pair [13-17]. The approaches differ in their predictions for the $Q^{2}$ dependence of the cross sections for excited vector-meson states and their ratio to their ground state.

The only radially excited $2 S$ triplet $q \bar{q}$ state studied at HERA so far has been the $\psi(2 S)$ state [18]. In this study, only the photoproduction reaction was investigated and the low cross-section ratio of $\psi(2 S)$ to the ground-state $J / \psi$ supported the existence of a suppression effect, expected if a node in the $\psi(2 S)$ wave-function is present.

Other excited vector-meson states, in particular those consisting of light quarks, can be used to study the effect caused by changing the scanning size. Exclusive $\pi^{+} \pi^{-}$production has been measured previously in the annihilation process $e^{+} e^{-} \rightarrow \pi^{+} \pi^{-}$[19], as well as in photoproduction [20]. The $\pi^{+} \pi^{-}$mass distribution shows a complex structure in the mass range 1-2 GeV. Evidence for two excited vector-meson states has been established [21, 22]; the $\rho^{\prime}(1450)$ is assumed to be predominantly a radially excited $2 S$ state and the $\rho^{\prime \prime}(1700)$ is an orbitally excited $2 D$ state, with some mixture of the $S$ and $D$ waves [23]. In addition there is also the $\rho_{3}(1690)$ spin-3 meson [24] which has a $\pi \pi$ decay mode. The two-pion decay mode of these resonances is related $[25,26]$ to the pion electromagnetic form factor, $F_{\pi}\left(M_{\pi \pi}\right)$.

In this paper, a study of exclusive electroproduction of two pions,

$\gamma^{*} p \rightarrow \pi^{+} \pi^{-} p$

is presented in the two-pion mass range $0.4<M_{\pi \pi}<$ $2.5 \mathrm{GeV}$, in the kinematic range $2<Q^{2}<80 \mathrm{GeV}^{2}, 32<$ $W<180 \mathrm{GeV}$ and $|t|<0.6 \mathrm{GeV}^{2}$, where $t$ is the squared four-momentum transfer at the proton vertex. The $M_{\pi \pi}$ system consists of a resonance part and a non-resonant background. The resonances are described by the pion form factor. The contributions of the three vector-mesons $\rho, \rho^{\prime}$ and

\footnotetext{
${ }^{\text {al }}$ Also at Łódź University, Poland

${ }^{a m}$ Member of Łódź University, Poland

${ }^{a n}$ Now at Department of Physics, Stockholm University, Stockholm, Sweden

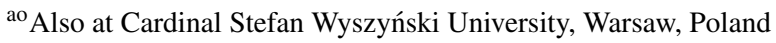

$\rho^{\prime \prime}$ are extracted and their relative rates as a function of $Q^{2}$ are discussed in terms of QCD expectations.

\section{The pion form factor}

The two-pion invariant-mass distribution of (1), after subtraction of the non-resonant background, ${ }^{1}$ can be related to the pion electromagnetic form factor, $F_{\pi}\left(M_{\pi \pi}\right)$, through the following relation $[25,26]$ :

$$
\frac{d N\left(M_{\pi \pi}\right)}{d M_{\pi \pi}} \propto\left|F_{\pi}\left(M_{\pi \pi}\right)\right|^{2}
$$

There are several parameterizations of the pion form factor usually used for fitting the $\pi^{+} \pi^{-}$mass distribution; the Kuhn-Santamaria (KS) [27], the Gounaris-Sakurai (GS) [28] and the Hidden Local Symmetry (HLS) [29, 30] parameterizations. In this paper, results based on the KS parameterization are presented.

In the mass range $M_{\pi \pi}<2.5 \mathrm{GeV}$, the KS parameterization of the pion form factor includes contributions from the $\rho(770), \rho^{\prime}(1450)$ and $\rho^{\prime \prime}(1700)$ resonances, ${ }^{2}$

$F_{\pi}\left(M_{\pi \pi}\right)=\frac{B W_{\rho}\left(M_{\pi \pi}\right)+\beta B W_{\rho^{\prime}}\left(M_{\pi \pi}\right)+\gamma B W_{\rho^{\prime \prime}}\left(M_{\pi \pi}\right)}{1+\beta+\gamma}$.

Here $\beta$ and $\gamma$ are relative amplitudes and $B W_{V}$ is the BreitWigner distribution which has the form

$B W_{V}\left(M_{\pi \pi}\right)=\frac{M_{V}^{2}}{M_{V}^{2}-M_{\pi \pi}^{2}-i M_{V} \Gamma_{V}\left(M_{\pi \pi}\right)}$,

where $M_{V}$ and $\Gamma_{V}\left(M_{\pi \pi}\right)$ are the vector-meson mass and momentum-dependent width, respectively. The latter has the form

$\Gamma_{V}\left(M_{\pi \pi}\right)=\Gamma_{V}\left[\frac{p_{\pi}\left(M_{\pi \pi}\right)}{p_{\pi}\left(M_{V}\right)}\right]^{3}\left[\frac{M_{V}^{2}}{M_{\pi \pi}^{2}}\right]$,

where $\Gamma_{V}$ is the width of the $V$ meson at $M_{\pi \pi}=M_{V}$, $p_{\pi}\left(M_{\pi \pi}\right)=1 / 2 \sqrt{M_{\pi \pi}^{2}-4 M_{\pi}^{2}}$ is the pion momentum in the $\pi^{+} \pi^{-}$centre-of-mass frame, $p_{\pi}\left(M_{V}\right)$ is the pion momentum in the $V$-meson rest frame, and $M_{\pi}$ is the pion mass.

\footnotetext{
${ }^{1}$ This is assumed not to interfere with the resonance signal.

${ }^{2}$ This analysis cannot distinguish between $\rho_{3}(1690)$ and $\rho^{\prime \prime}(1700)$. Theoretical calculations estimate the contribution of $\rho_{3}(1690)$ to be either one order of magnitude [12] or 2-5 times [31] smaller than that of the $\rho^{\prime \prime}(1700)$.
} 


\section{Experimental set-up}

The analyzed data were collected with the ZEUS detector at the HERA collider in the years 1998-2000, when $920 \mathrm{GeV}$ protons collided with $27.5 \mathrm{GeV}$ electrons or positrons. The sample used for this study corresponds to $81.7 \mathrm{pb}^{-1}$ of which $65.0 \mathrm{pb}^{-1}$ were collected with an $e^{+}$and the rest with an $e^{-}$beam. ${ }^{3}$

A detailed description of the ZEUS detector can be found elsewhere [32]. A brief outline of the components that are most relevant for this analysis is given below.

The charged particles were tracked in the central tracking detector (CTD) [33-35] which operated in a magnetic field of $1.43 \mathrm{~T}$ provided by a thin superconducting solenoid. The CTD consisted of 72 cylindrical drift chamber layers, organized in nine superlayers covering the polar-angle ${ }^{4}$ region $15^{\circ}<\theta<164^{\circ}$. The transverse-momentum resolution for full-length tracks was $\sigma\left(p_{T}\right) / p_{T}=0.0058 p_{T} \oplus 0.0065 \oplus$ $0.0014 / p_{T}$, with $p_{T}$ in $\mathrm{GeV}$.

The scattered electron was identified in the high-resolution uranium-scintillator calorimeter (CAL) [36-39] which covered $99.7 \%$ of the total solid angle and consisted of three parts: the forward (FCAL), the barrel (BCAL) and the rear (RCAL) calorimeters. Each part was subdivided transversely into towers and longitudinally into one electromagnetic section (EMC) and either one (in RCAL) or two (in BCAL and FCAL) hadronic sections (HAC). The CAL energy resolution, as measured under test-beam conditions, was $\sigma(E) / E=0.18 / \sqrt{E}$ for electrons and $\sigma(E) / E=$ $0.35 / \sqrt{E}$ for hadrons, with $E$ in $\mathrm{GeV}$.

The position of the scattered electron was determined by combining information from the CAL, the small-angle rear tracking detector [40] and the hadron-electron separator [41].

The luminosity was measured from the rate of the bremsstrahlung process $e p \rightarrow e \gamma p$. The photon was measured in a lead-scintillator calorimeter [42-44] placed in the HERA tunnel at $Z=-107 \mathrm{~m}$.

\section{Data selection and reconstruction}

The online event selection required an electron candidate in the CAL, along with the detection of at least one and not more than six tracks in the CTD.

In the offline selection, the following further requirements were imposed:

\footnotetext{
${ }^{3}$ From now on, electrons and positrons will be both referred to as electrons in this paper.

${ }^{4}$ The ZEUS coordinate system is a right-handed Cartesian system, with the $Z$ axis pointing in the proton beam direction, referred to as the "forward direction", and the $X$ axis pointing left towards the centre of HERA. The coordinate origin is at the nominal interaction point. The polar angle, $\theta$, is measured with respect to the proton beam direction.
}

- the presence of a scattered electron, with energy in the CAL greater that $10 \mathrm{GeV}$ and with an impact point on the face of the RCAL outside a rectangular area of $26.4 \times$ $16 \mathrm{~cm}^{2}$ in the $X-Y$ plane;

- the $Z$ coordinate of the interaction vertex was within $\pm 50 \mathrm{~cm}$ of the nominal interaction point;

- in addition to the scattered electron, the presence of exactly two oppositely charged tracks. Both tracks have to be associated with the reconstructed vertex, each having pseudorapidity $|\eta|$ less than 1.75 and transverse momentum greater that $150 \mathrm{MeV}$. This ensures high reconstruction efficiency and excellent momentum resolution in the CTD. These tracks were treated in the following analysis as a $\pi^{+} \pi^{-}$pair;

- $E-P_{Z}>45 \mathrm{GeV}$, where $E-P_{Z}=\sum_{i}\left(E_{i}-P_{Z_{i}}\right)$ and the summation is over the energy $E_{i}$ and longitudinal momentum $P_{Z_{i}}$ of the final-state electron and pions. This cut excludes events with high-energy photons radiated in the initial state;

- events with any energy deposit larger than $300 \mathrm{MeV}$ in the CAL, not associated with the pion tracks (so-called 'unmatched islands'), were rejected.

The following kinematic variables are used to describe the exclusive production of a $\pi^{+} \pi^{-}$pair:

- $Q^{2}$, the four-momentum squared of the virtual photon;

- $W^{2}$, the squared centre-of-mass energy of the photonproton system;

- $M_{\pi \pi}$, the invariant mass of the two pions;

- $t$, the squared four-momentum transfer at the proton vertex;

- $\Phi_{h}$, the angle between the $\pi^{+} \pi^{-}$production plane and the positron scattering plane in the $\gamma^{*} p$ centre-of-mass frame;

- $\theta_{h}$ and $\phi_{h}$, the polar and azimuthal angles of the positively charged pion in the $s$-channel helicity frame [45] of the $\pi^{+} \pi^{-}$.

The kinematic variables were reconstructed using the socalled 'constrained' method [46], which uses the momenta of the decay particles measured in the CTD and the reconstructed polar and azimuthal angles of the scattered electron. The analysis was restricted to the kinematic region $2<Q^{2}<80 \mathrm{GeV}^{2}, 32<W<180 \mathrm{GeV},|t| \leq 0.6 \mathrm{GeV}^{2}$ and $0.4<M_{\pi \pi}<2.5 \mathrm{GeV}$. The lower mass range excludes reflections from the $\phi \rightarrow K^{+} K^{-}$decays and the upper limit excludes the $J / \psi \rightarrow \mu^{+} \mu^{-}, e^{+} e^{-}$decays with its radiative tail.

The above selection yielded 63517 events for this analysis.

The above cuts do not eliminate events in which the proton dissociates into a low-mass final state, the products of which disappear down the beam pipe. This contribution, estimated [2] to be about $20 \%$ in the range of this analysis, 
was found to be $Q^{2}$ and $W$ independent. Its presence does not affect the conclusions of this analysis.

\section{Monte Carlo simulation}

The program Zeusvm [47] interfaced to HeraCLES4.4 [48] was used. The effective distributions of $Q^{2}, W$ and $|t|$ were parameterized to reproduce the data. The mass and angular distributions were generated uniformly and the MC events were then iteratively reweighted using the results of the analysis.

The generated events were passed through a full simulation of the ZEUS detector based on GEANT 3.21 [49] and processed through the same chain of selection and reconstruction procedures as the data, accounting for trigger as well as detector acceptance and smearing effects. The number of simulated events after reconstruction was approximately seven times greater than the number of reconstructed data events.

A detailed comparison between the data and the ZEUSVM MC distributions for the mass range $0.65<M_{\pi \pi}<1.1 \mathrm{GeV}$ has been presented elsewhere [2]. Some examples for the mass range $1.1<M_{\pi \pi}<2.1 \mathrm{GeV}$ are shown here. The transverse momentum, $p_{T}$, of the $\pi^{+}$and the $\pi^{-}$particles for different ranges of $Q^{2}$ and $M_{\pi \pi}$ are presented in Fig. 1. Figure 2 shows the $Q^{2}, W,|t|, \cos \theta_{h}, \phi_{h}$, and $\Phi_{h}$ distributions for events selected within the mass ranges $1.1<M_{\pi \pi}<1.6 \mathrm{GeV}$, while Fig. 3 shows those distributions for the mass range $1.6<M_{\pi \pi}<2.1 \mathrm{GeV}$. All measured distributions are well described by the MC simulations.

\section{The $\pi \pi$ mass fit}

The $\pi^{+} \pi^{-}$mass distribution, after acceptance correction determined from the above MC simulation, is shown in Fig. 4. A clear peak is seen in the $\rho$ mass range. A small shoulder is apparent around $1.3 \mathrm{GeV}$ and a secondary peak at about $1.8 \mathrm{GeV}$.

The two-pion invariant-mass distribution was fitted, using the least-square method [50], as a sum of two terms,

$$
\frac{d N\left(M_{\pi \pi}\right)}{d M_{\pi \pi}}=A\left(1-\frac{4 M_{\pi}^{2}}{M_{\pi \pi}^{2}}\right)\left[\left|F_{\pi}\left(M_{\pi \pi}\right)\right|^{2}+B\left(\frac{M_{0}}{M_{\pi}}\right)^{n}\right],
$$

where $A$ is an overall normalization constant. The second term is a parameterization of the non-resonant background, with constant parameters $B, n$ and $M_{0}=1 \mathrm{GeV}$. The other parameters, the masses and widths of the three resonances and their relative contributions $\beta$ and $\gamma$, enter through the

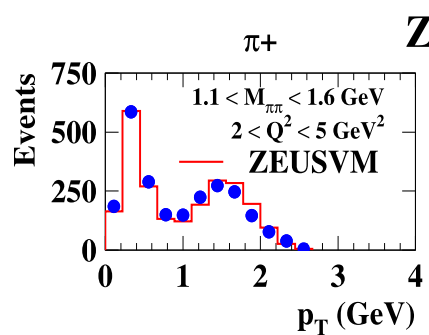

ZEUS $\pi$ -
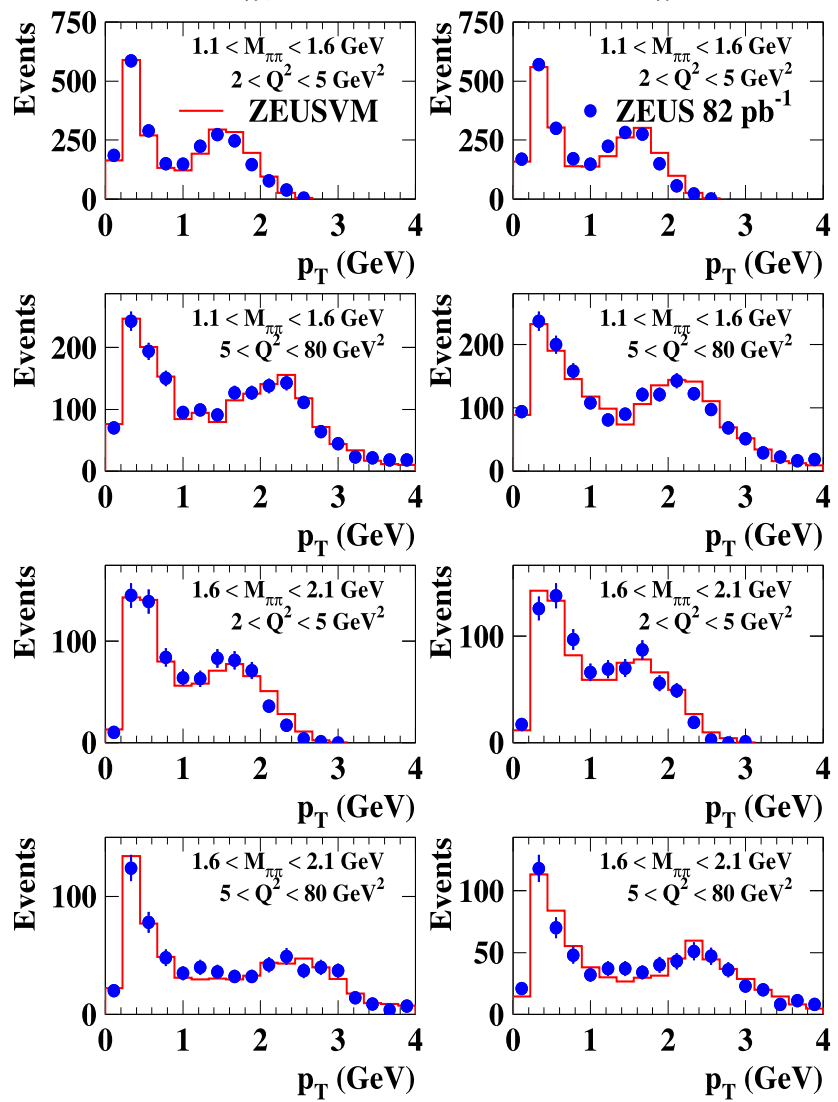

Fig. 1 Comparison between the data and the Zeusvm MC distributions for the transverse momentum, $p_{T}$, of $\pi^{+}$and $\pi^{-}$particles for different ranges of $Q^{2}$ and $M_{\pi \pi}$ as indicated in the figure. The MC distributions are normalized to the data

pion form factor, $F_{\pi}$ (see (3)). The fit, which includes 11 parameters, gives a good description of the data $\left(\chi^{2} / \mathrm{ndf}=\right.$ $28.8 / 24=1.2)$. The result of the fit is shown in Fig. 4 together with the contribution of each of the two terms of (6). The $\rho$ and the $\rho^{\prime \prime}$ signals are clearly visible. The negative interference between all the resonances results in the $\rho^{\prime}$ signal appearing as a shoulder. To illustrate this better, the same data and fit are shown in Fig. 5 on a linear scale and limited to $M_{\pi \pi}>1.2 \mathrm{GeV}$, with separate contributions from the background, the three resonant amplitudes as well as their total interference term.

The fit parameters are listed in Table 1. Also listed are the mass and width parameters from the Particle Data Group (PDG) [51]. The masses and widths of the $\rho$ and the $\rho^{\prime \prime}$ as well as the width of the $\rho^{\prime}$ agree with those listed in the PDG, while there is about $100 \mathrm{MeV}$ difference between the PDG value and the fitted mass of the $\rho^{\prime}$. It should however be noted that the value quoted by PDG is an average over many measurements having a large spread (1265 \pm 75 up to $1424 \pm 25 \mathrm{MeV}$ for the $\pi \pi$ decay mode) in this mass range.

The measured negative value of $\beta$ and positive value of $\gamma$ implies that the relative signs of the amplitudes of the 
ZEUS
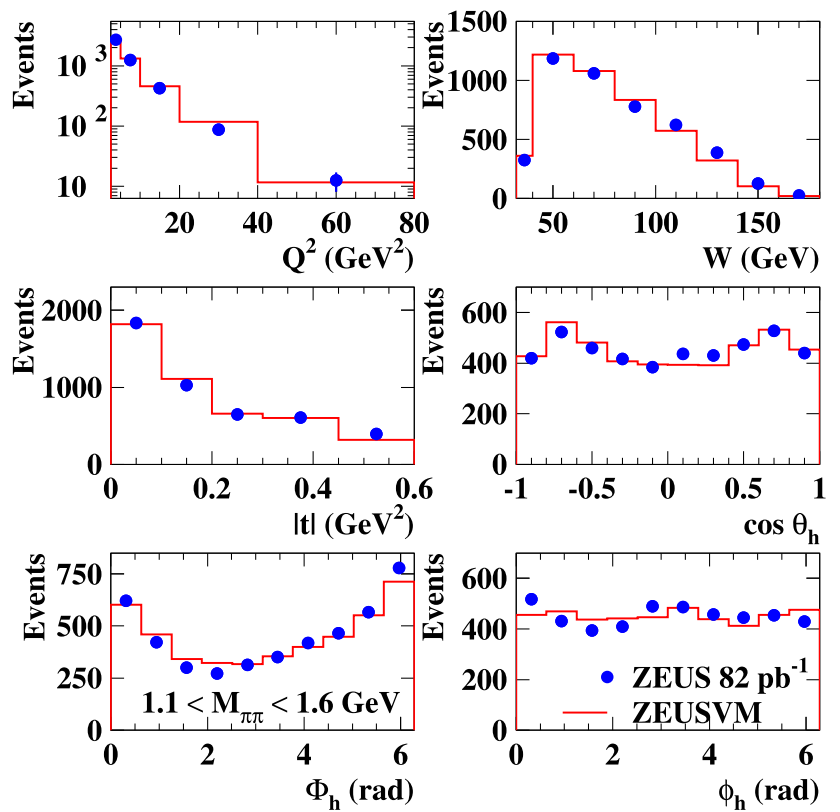

Fig. 2 Comparison between the data and the ZEusvm MC distributions for $Q^{2}, W,|t|, \cos \theta_{h}, \Phi_{h}$ and $\phi_{h}$ for events within mass range $1.1<M_{\pi \pi}<1.6 \mathrm{GeV}$. The MC distributions are normalized to the data

\section{ZEUS}
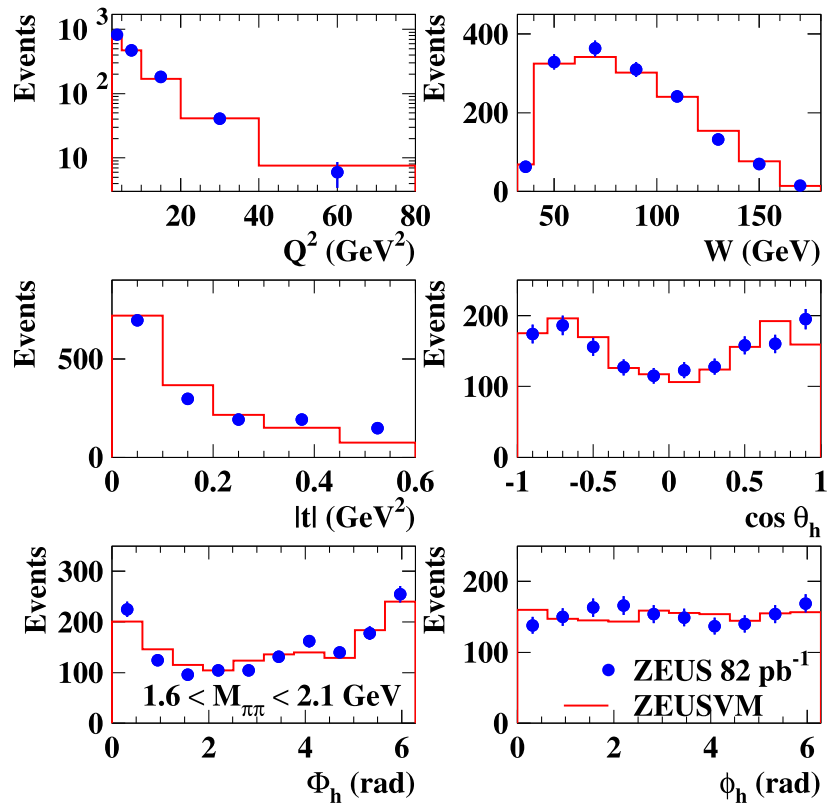

Fig. 3 Comparison between the data and the ZEUsvm MC distributions for $Q^{2}, W,|t|, \cos \theta_{h}, \Phi_{h}$ and $\phi_{h}$ for events within mass range $1.6<M_{\pi \pi}<2.1 \mathrm{GeV}$. The MC distributions are normalized to the data

three resonances $\rho, \rho^{\prime}$ and $\rho^{\prime \prime}$ are,,+-+ , respectively. A similar pattern was observed in $e^{+} e^{-} \rightarrow \pi^{+} \pi^{-}$and $\tau$ decay experiments [53-61], which also showed a dip in
ZEUS

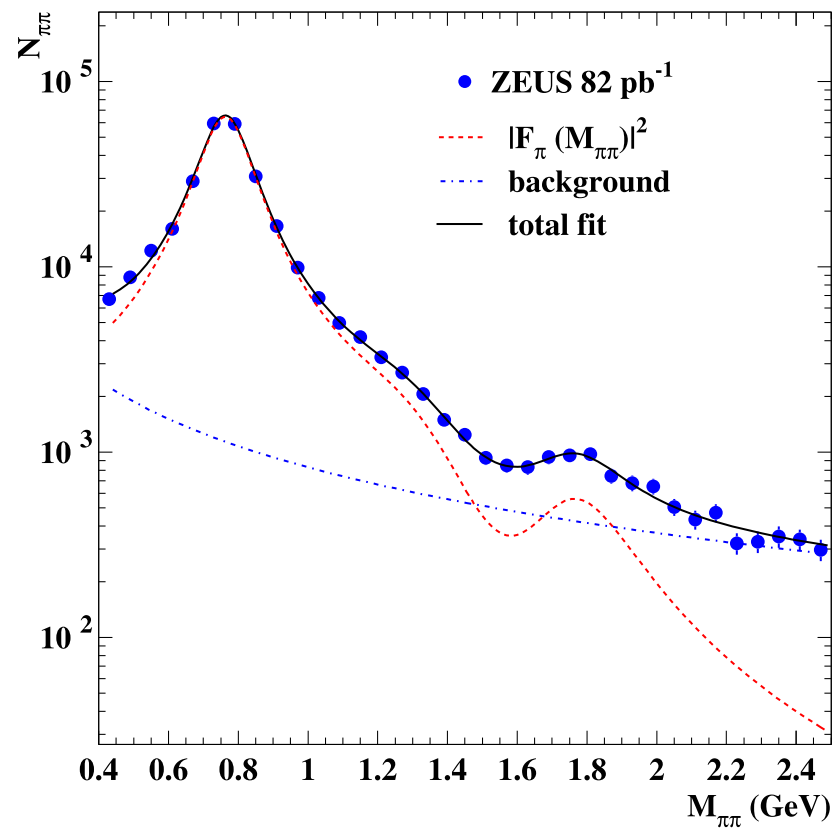

Fig. 4 The two-pion invariant-mass distribution, $M_{\pi \pi}$, where $N_{\pi \pi}$ is the acceptance-corrected number of events in each bin of $60 \mathrm{MeV}$. The dots are the data and the full line is the result of a fit using the Kuhn-Santamaria parameterization. The dashed line is the result of the pion form factor normalized to the data and the dash-dotted line denotes the background contribution

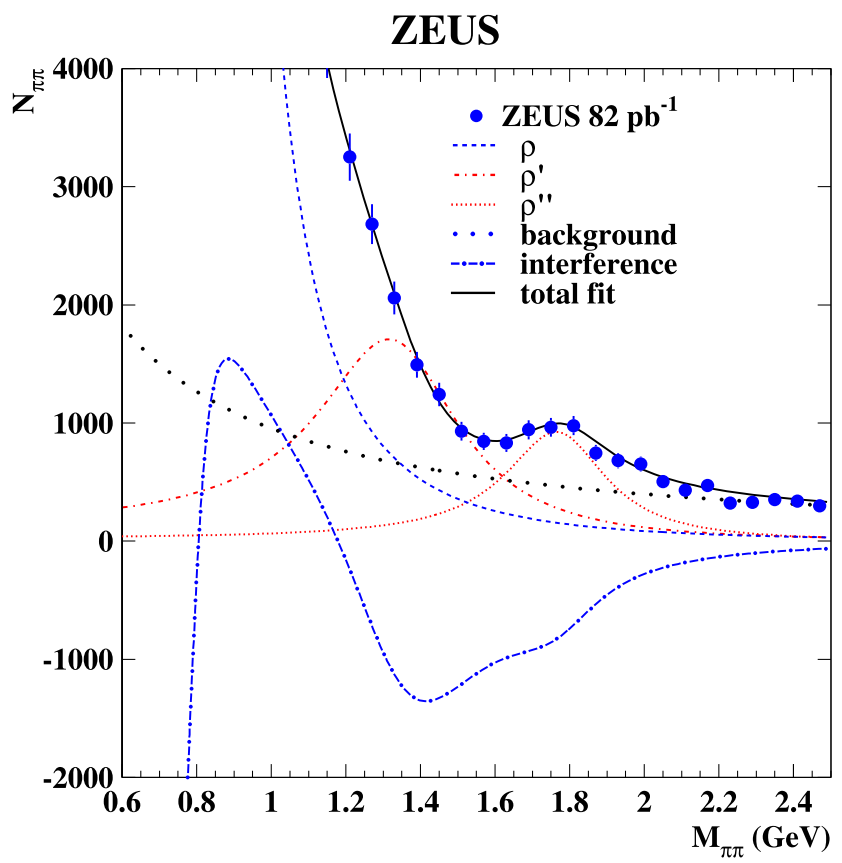

Fig. 5 The two-pion invariant-mass distribution, $M_{\pi \pi}$, where $N_{\pi \pi}$ is the acceptance corrected number of events in each bin of $60 \mathrm{MeV}$. The dots are the data and the full line is the result of a fit using the Kuhn-Santamaria parameterization. The contributions of the three resonances $\rho, \rho^{\prime}$ and $\rho^{\prime \prime}$ are shown as dashed, dash-dotted and dotted lines, respectively. The sum of their interferences is shown by the longdash-dotted line. The background is presented as the sparse dotted line 
the mass range around $1.6 \mathrm{GeV}$, resulting from destructive interference. There is a single experiment where a constructive interference was obtained around $1.6 \mathrm{GeV}$, namely $\gamma p \rightarrow \pi^{+} \pi^{-} p$ [20], a result which is not understood [15].
In the mass fits above it was assumed that the relative amplitudes $\beta$ and $\gamma$ are real. In order to test this assumption, the fit was repeated allowing them to be complex. The pion form factor was re-written in the form

$F_{\pi}\left(M_{\pi \pi}\right)=\frac{B W_{\rho}\left(M_{\pi \pi}\right)+\beta_{0} \cdot \exp \left(i \Phi_{12}\right) B W_{\rho^{\prime}}\left(M_{\pi \pi}\right)+\gamma_{0} \cdot \exp \left(i \Phi_{13}\right) B W_{\rho^{\prime \prime}}\left(M_{\pi \pi}\right)}{1+\beta_{0}+\gamma_{0}}$,

where $\beta_{0}$ and $\gamma_{0}$ are real numbers and two additional fit parameters, $\Phi_{12}$ and $\Phi_{13}$, are the corresponding phase shifts. The value of the phase-shifts obtained from the fit were $\Phi_{12}=3.2 \pm 0.2 \mathrm{rad}$ and $\Phi_{13}=0.1 \pm 0.2 \mathrm{rad}$, supporting the assumption of the real nature of the relative amplitudes.

\section{Systematic uncertainties}

The systematic uncertainties of the fit parameters were evaluated by varying the selection cuts and the MC simulation parameters. Motivation for the variation in cuts used below can be found in a previous ZEUS analysis [2]. The following selection cuts were varied:

- the $E-P_{Z}$ cut was changed within the resolution of $\pm 3 \mathrm{GeV}$;

- the $p_{T}$ threshold for the pion tracks (default $0.15 \mathrm{GeV}$ ) was increased to $0.2 \mathrm{GeV}$ and the $|\eta|$ cut on the two pion tracks was changed (default 1.75) by \pm 0.25 ;

- the required maximum distance of closest approach of the two extrapolated pion tracks to the matched island in the CAL was changed from $30 \mathrm{~cm}$ to $20 \mathrm{~cm}$;

- the $Z$-vertex cut was varied by $\pm 10 \mathrm{~cm}$;

Table 1 Fit parameters obtained using the $F_{\pi}\left(M_{\pi \pi}\right)$ parameterization. Masses and widths are in $\mathrm{MeV}$. The first uncertainty is statistical, the second systematic. Also shown are the masses and widths from the PDG [51]

\begin{tabular}{lll}
\hline Parameter & ZEUS & PDG \\
\hline$M_{\rho}(\mathrm{MeV})$ & $771 \pm 2_{-1}^{+2}$ & $775.49 \pm 0.34$ \\
$\Gamma_{\rho}(\mathrm{MeV})$ & $155 \pm 5 \pm 2$ & $149.1 \pm 0.8$ \\
$\beta$ & $-0.27 \pm 0.02 \pm 0.02$ & \\
$M_{\rho^{\prime}}(\mathrm{MeV})$ & $1350 \pm 20_{-30}^{+20}$ & $1465 \pm 25$ \\
$\Gamma_{\rho^{\prime}}(\mathrm{MeV})$ & $460 \pm 30_{-45}^{+40}$ & $400 \pm 60$ \\
$\gamma$ & $0.10 \pm 0.02_{-0.01}^{+0.02}$ & \\
$M_{\rho^{\prime \prime}}(\mathrm{MeV})$ & $1780 \pm 20_{-20}^{+15}$ & $1720 \pm 20$ \\
$\Gamma_{\rho^{\prime \prime}}(\mathrm{MeV})$ & $310 \pm 30_{-35}^{+25}$ & $250 \pm 100$ \\
$B$ & $0.41 \pm 0.03 \pm 0.07$ & \\
$n$ & $1.30 \pm 0.06_{-0.13}^{+0.18}$ & \\
\hline
\end{tabular}

- the energy threshold for an unmatched island (elasticity cut) was changed by $\pm 50 \mathrm{MeV}$;

- the bin size in the fitted mass distribution (default $60 \mathrm{MeV}$ ) was varied by $\pm 20 \mathrm{MeV}$;

- the mass range was narrowed to $0.5<M_{\pi \pi}<2.3 \mathrm{GeV}$;

- the $|t|$ cut was varied by $\pm 0.1 \mathrm{GeV}^{2}$;

- the $W$ range was changed to $35<W<190 \mathrm{GeV}$;

- the $\cos \theta_{h}$ range was changed to $\left|\cos \theta_{h}\right|<0.9$;

- the $W^{\delta}$ dependence in the MC was varied by changing the $Q^{2}$-dependent $\delta$ value by \pm 0.03 ;

- the exponential $t$ distribution in the MC was reweighted by changing the nominal $Q^{2}$-dependent slope parameter $b$ by $\pm 0.5 \mathrm{GeV}^{-2}$;

- the exponent of the $Q^{2}$ distribution parameterization in the MC was changed by \pm 0.05 .

The largest variations were observed for $\gamma, \Gamma\left(\rho^{\prime \prime}\right)$ and $\beta$. The value of $\Gamma\left(\rho^{\prime \prime}\right)$ changes by $7 \%$ when the elasticity cut is varied. The restriction of the phase space in the fitted mass range leads to a change of the value of $\beta$ by $-5.2 \%$ while for $\gamma$, restricting the $\left|\cos \theta_{h}\right|$ range leads to a change of $-8 \%$. In addition, another form of background in (6), with an added exponential term, was investigated. It gave a very similar result in the mass range of this analysis and therefore no additional uncertainty was assigned to the form of the fitted mass curve.

All the systematic uncertainties were added in quadrature. The combined systematic uncertainties are included in Table 1.

\section{Decay angular distributions}

Decay angular distributions can be used to determine the spin density-matrix elements of a resonance [45, 52]. In the present case we study three resonances, all in a $J^{P}=1^{-}$ state. However, the decay angular distribution in a given mass bin is affected by the background contribution which does not necessarily have the same quantum numbers as the resonance. Given the above, only the distribution of the polar angle $\theta_{h}$, defined as the polar angle of the positively charged pion in the helicity frame, was studied. 
The distribution of $\cos \theta_{h}$ is shown in Fig. 6 for different mass bins; its shape is clearly mass dependent. In order to study the mass dependence further, the angular distribution of the polar helicity angle, $W\left(\cos \theta_{h}\right)$ was parameterized as

$W\left(\cos \theta_{h}\right) \propto\left[1-r+(3 r-1) \cos ^{2} \theta_{h}\right]$,

and fitted to the data. The mass dependence of the resulting parameter $r$ is shown in Fig. 7. In the mass range $M_{\pi \pi}<$ $1.1 \mathrm{GeV}, r$ shows the dependence seen for the $r_{00}^{04}$ density matrix in the $\rho$ region [2]. Indeed this region is dominated by exclusive production of $\rho$ and therefore $r=r_{00}^{04}$. In that case, $r$ can be interpreted as $\sigma_{L} / \sigma_{\mathrm{tot}}$, assuming $s$-channel helicity conservation (SCHC). Here $\sigma_{L}$ is the cross section for producing $\rho$ by a longitudinally polarized photon, and $\sigma_{\text {tot }}=\sigma_{L}+\sigma_{T}$, with $\sigma_{T}$ the production cross section by transversely polarized photons. The results shown here for the $\rho$ region are in excellent agreement with the values given in an earlier ZEUS paper [2].
The structure seen for $M_{\pi \pi}>1.1 \mathrm{GeV}$ is not easy to interpret, however the dip observed around $1.3 \mathrm{GeV}$ and the enhancement at $1.6 \mathrm{GeV}$ seem to follow the location of the resonances determined from the mass distribution.

\section{$9 Q^{2}$ dependence of the pion form factor}

The $Q^{2}$ dependence of the relative amplitudes was determined by performing the fit to $M_{\pi \pi}$ in three $Q^{2}$ regions, 2-5, 5-10 and 10-80 $\mathrm{GeV}^{2}$. The masses and widths of the three resonances were fixed to the values found in the overall fit and listed in Table 1. The results are shown in Fig. 8. A reasonable description of the data is achieved in all three $Q^{2}$ regions. The corresponding values of $\beta$ and $\gamma$ are given in Table 2. The absolute value of $\beta$ increases with $Q^{2}$ while the value of $\gamma$ is consistent with no $Q^{2}$ dependence, within large uncertainties.
Fig. 6 The

acceptance-corrected $\cos \theta_{h}$ distribution for different $M_{\pi \pi}$ intervals, with the mean mass values indicated in the figure. The lines represent fits to the data as discussed in the text
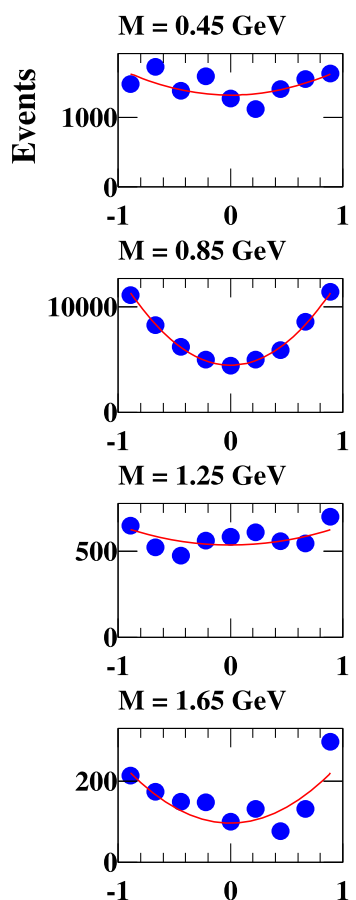

$\mathrm{M}=2.05 \mathrm{GeV}$
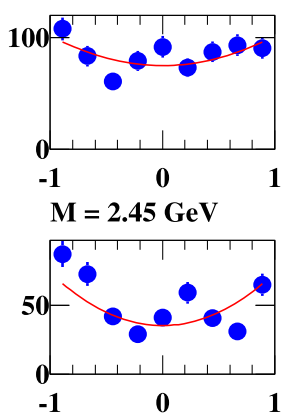

\section{ZEUS}
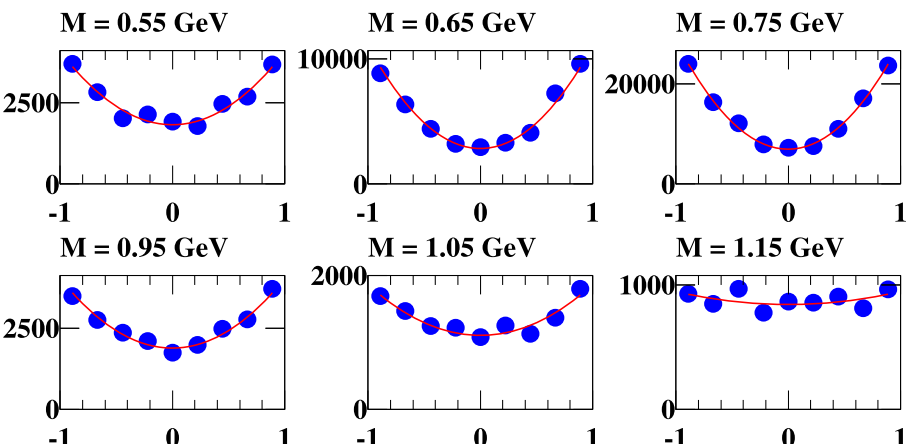

2000
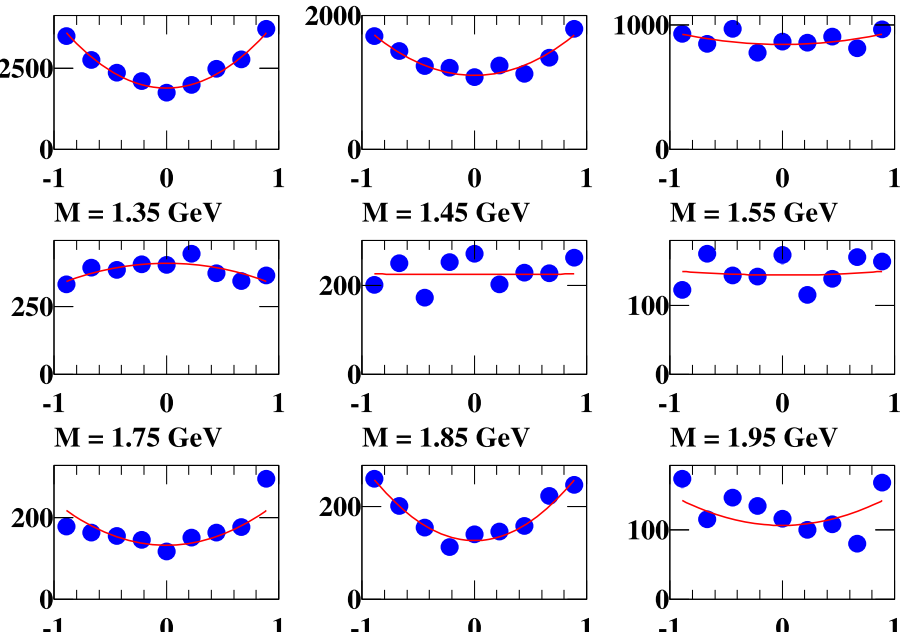

$\mathrm{M}=1.85 \mathrm{GeV}$

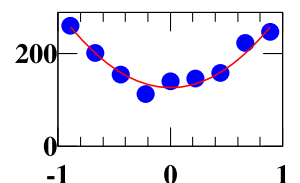

$\mathrm{M}=2.15 \mathrm{GeV}$

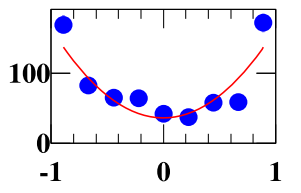

$\mathrm{M}=2.25 \mathrm{GeV}$

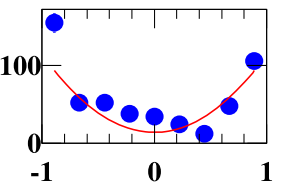

10

$\mathrm{M}=1.95 \mathrm{GeV}$

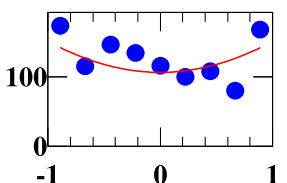

$\mathrm{M}=2.35 \mathrm{GeV}$

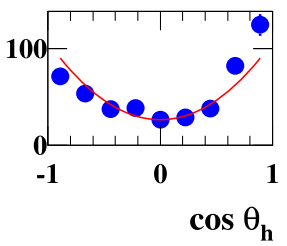

- ZEUS 82 pb $^{-1}$ 
Table 2 The $Q^{2}$ dependence of the $\beta$ and $\gamma$ parameters. Masses and widths are fixed to the values given in Table 1 . The first uncertainty is statistical, the second systematic

\begin{tabular}{llll}
\hline$Q^{2}\left(\mathrm{GeV}^{2}\right)$ & $2-5$ & $5-10$ & $10-80$ \\
\hline$\beta$ & $-0.249 \pm 0.008_{-0.003}^{+0.005}$ & $-0.282 \pm 0.008_{-0.008}^{+0.005}$ & $-0.35 \pm 0.02 \pm 0.01$ \\
$\gamma$ & $0.100 \pm 0.009 \pm 0.003$ & $0.098 \pm 0.012_{-0.003}^{+0.005}$ & $0.118 \pm 0.022_{-0.006}^{+0.008}$ \\
\hline
\end{tabular}

\section{ZEUS}

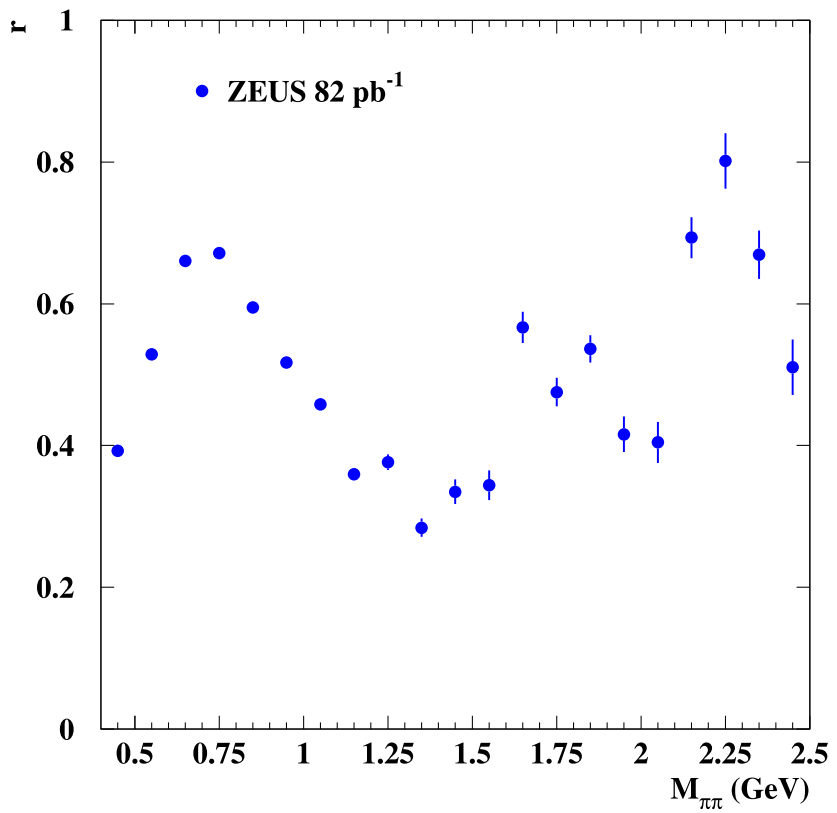

Fig. 7 The fitted parameter $r$ as a function of the two-pion invariant mass, $M_{\pi \pi}$. Only statistical uncertainties are shown

Figure 9 shows the curves representing the pion form factor, $\left|F_{\pi}\left(M_{\pi \pi}\right)\right|^{2}$, as obtained in the present analysis for the three $Q^{2}$ ranges: $2-5,5-10,10-80 \mathrm{GeV}^{2}$. Also shown are results obtained in the time-like regime from the reaction $e^{+} e^{-} \rightarrow \pi^{+} \pi^{-}$. In general, the features of the $\left|F_{\pi}\left(M_{\pi \pi}\right)\right|^{2}$ distribution observed here are also observed in $e^{+} e^{-}$, i.e., the prominent $\rho$ peak, a shoulder around the $\rho^{\prime}$ and a dip followed by an enhancement in the $\rho^{\prime \prime}$ region. Above the $\rho$ region, where the interference between the $\rho^{\prime}$ and the $\rho^{\prime \prime}$ starts to dominate, there is a dependence of $\left|F_{\pi}\left(M_{\pi \pi}\right)\right|^{2}$ on $Q^{2}$, with the results from the lowest $Q^{2}$ range closest to those from $e^{+} e^{-}$. However, in the region of the $\rho$ peak, shown in Fig. 10, the pion form-factor $\left|F_{\pi}\left(M_{\pi}\right)\right|^{2}$ is highest at the highest $Q^{2}$, as in the $\rho^{\prime}-\rho^{\prime \prime}$ interference region, while the $e^{+} e^{-}$data are higher than those in the highest $Q^{2}$ range. They are equal within errors for $M_{\pi \pi}>1.8 \mathrm{GeV}$.

\section{Cross-section ratios as a function of $Q^{2}$}

The $Q^{2}$ dependence of the $\rho$ by itself is given elsewhere [2]. Since the $\pi \pi$ branching ratios of $\rho^{\prime}$ and $\rho^{\prime \prime}$ are poorly

\section{ZEUS}
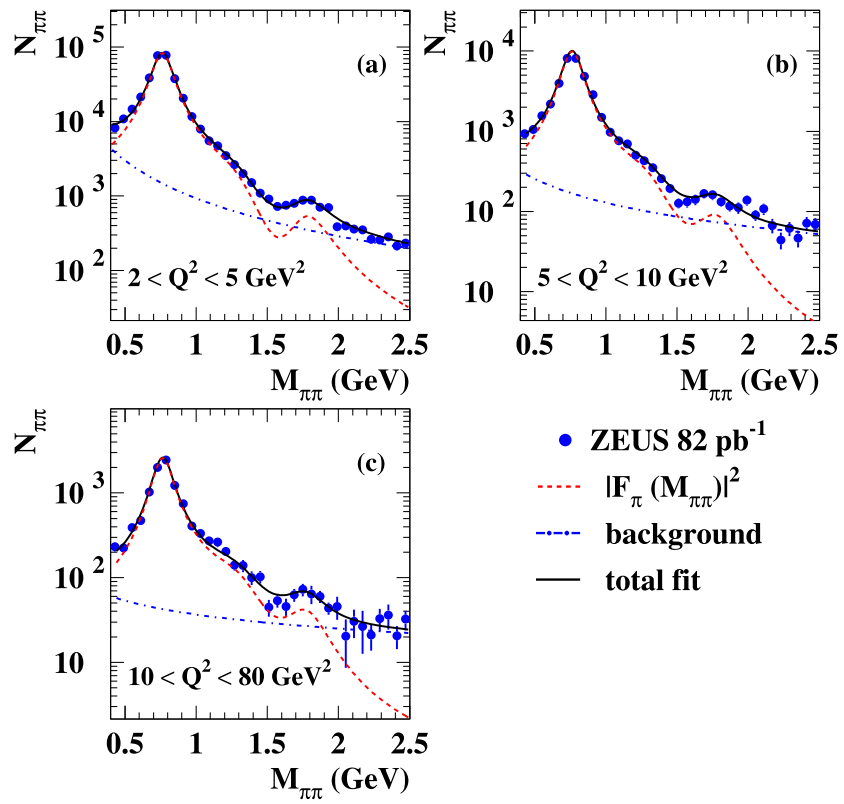

- ZEUS 82 pb $^{-1}$

$\left|\mathbf{F}_{\pi}\left(\mathbf{M}_{\pi \pi}\right)\right|^{2}$

-.--- background

- total fit

Fig. 8 The two-pion invariant-mass distribution, $M_{\pi \pi}$, where $N_{\pi \pi}$ is the acceptance-corrected number of events in each bin of $60 \mathrm{MeV}$, for three regions of $Q^{2}$, as denoted in the figure. The dots are the data and the full line is the result of a fit using the Kuhn-Santamaria parameterization. The dashed line is the result of the pion form factor normalized to the data and the dash-dotted line denotes the background contribution

known, the ratio $R_{V}$ defined as

$R_{V}=\frac{\sigma(V) \cdot B r(V \rightarrow \pi \pi)}{\sigma(\rho)}$,

has been measured, where $\sigma$ is the cross section for vectormeson production and $\operatorname{Br}(V \rightarrow \pi \pi)$ is the branching ratio of the vector meson $V\left(\rho^{\prime}, \rho^{\prime \prime}\right)$ into $\pi \pi$. The ratio $R_{V}$ may be directly determined from the results of the $M_{\pi \pi}$ mass fit,

$R_{\rho^{\prime}}=\beta^{2} \frac{I_{\rho^{\prime}}}{I_{\rho}}, \quad R_{\rho^{\prime \prime}}=\gamma^{2} \frac{I_{\rho^{\prime \prime}}}{I_{\rho}}$,

where

$I_{V}=\int_{2 M_{\pi}}^{M_{V}+5 \Gamma_{V}} d M_{\pi \pi}\left|B W_{V}\left(M_{\pi \pi}\right)\right|^{2}$,

and the integration is carried out over the range $2 M_{\pi}<$ $M_{\pi \pi}<M_{V}+5 \Gamma_{V}$. 


\section{ZEUS}

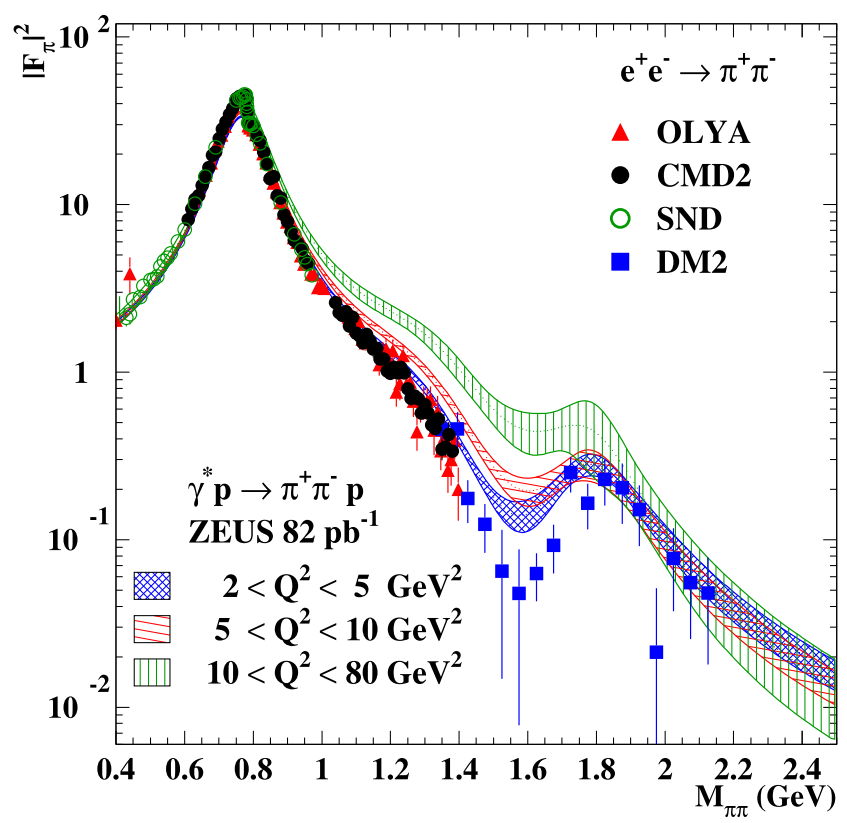

Fig. 9 The pion form factor squared, $\left|F_{\pi}\right|^{2}$, as a function of the $\pi^{+} \pi^{-}$invariant mass, $M_{\pi \pi}$, as obtained from the reaction $e^{+} e^{-} \rightarrow \pi^{+} \pi^{-}[19,53,54,56,57]$. The shaded bands represent the square of the pion form factor and its total uncertainty obtained in the present analysis for three ranges of $Q^{2}: 2-5 \mathrm{GeV}^{2}$ (crossed lines), $5-10 \mathrm{GeV}^{2}$ (horizontal lines) and $10-80 \mathrm{GeV}^{2}$ (vertical lines)

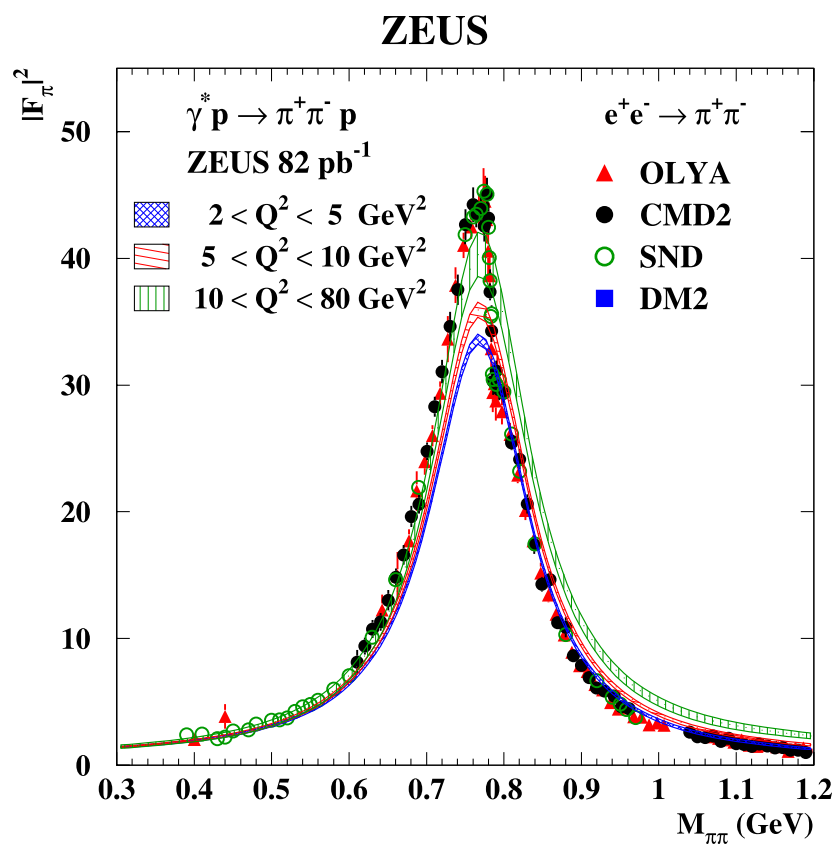

Fig. 10 The pion form factor squared, $\left|F_{\pi}\right|^{2}$, in the $\rho$ mass region, as a function of the $\pi^{+} \pi^{-}$invariant mass, $M_{\pi \pi}$, as obtained from the reaction $e^{+} e^{-} \rightarrow \pi^{+} \pi^{-}[19,53,54,56,57]$. The shaded bands represent the square of the pion form factor and its total uncertainty obtained in the present analysis for three ranges of $Q^{2}: 2-5 \mathrm{GeV}^{2}$ (crossed lines), $5-10 \mathrm{GeV}^{2}$ (horizontal lines) and $10-80 \mathrm{GeV}^{2}$ (vertical lines)
ZEUS

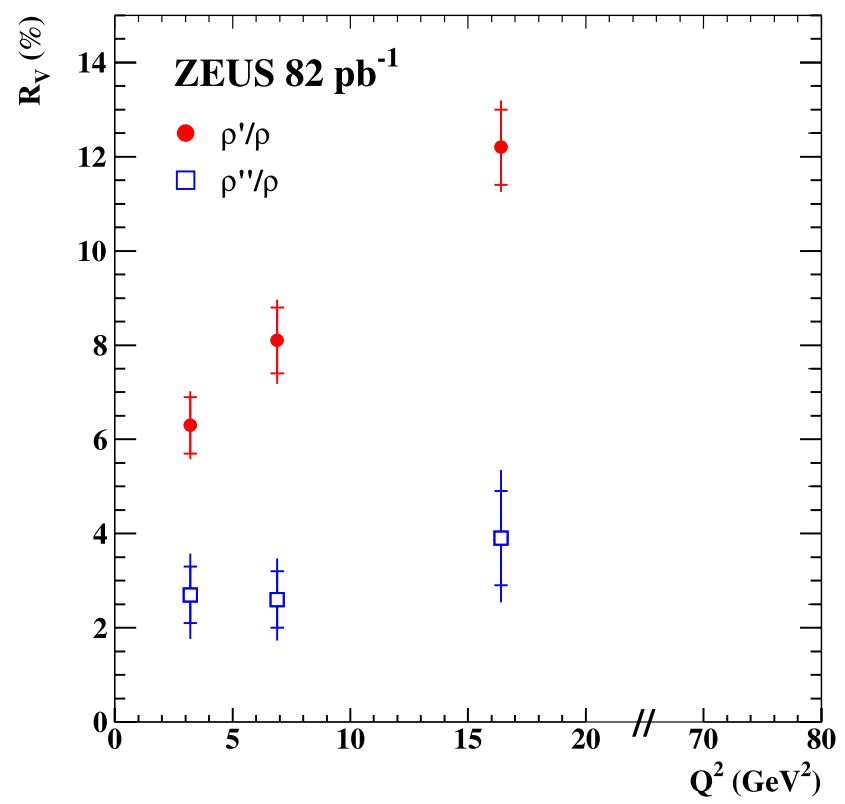

Fig. 11 The ratio $R_{V}$ as a function of $Q^{2}$ for $V=\rho^{\prime}$ (full circles) and $\rho^{\prime \prime}$ (open squares). The inner error bars indicate the statistical uncertainty, the outer error bars represent the statistical and systematic uncertainty added in quadrature

Figure 11 shows and Table 3 lists the ratio $R_{V}$ for $V=$ $\rho^{\prime}, \rho^{\prime \prime}$, as a function of $Q^{2}$. Owing to the large uncertainties of $R_{\rho^{\prime \prime}}$, no conclusion on its $Q^{2}$ behaviour can be deduced, whereas $R_{\rho^{\prime}}$ clearly increases with $Q^{2}$. This rise has been predicted by several models $[11,13,16,62,63]$. The suppression of the $2 S$ state $\left(\rho^{\prime}\right)$ is connected to a node effect which results in cancellations of contributions from different impact-parameter regions at lower $Q^{2}$, while at higher $Q^{2}$ the effect vanishes.

\section{Summary}

Exclusive two-pion electroproduction has been studied by ZEUS at HERA in the range $0.4<M_{\pi \pi}<2.5 \mathrm{GeV}, 2<$ $Q^{2}<80 \mathrm{GeV}^{2}, 32<W<180 \mathrm{GeV}$ and $|t| \leq 0.6 \mathrm{GeV}^{2}$. The mass distribution is well described by the pion electromagnetic form factor, $\left|F_{\pi}\left(M_{\pi \pi}\right)\right|^{2}$, which includes three resonances, $\rho, \rho^{\prime}(1450)$ and $\rho^{\prime \prime}(1700)$.

A $Q^{2}$ dependence of $\left|F_{\pi}\left(M_{\pi \pi}\right)\right|^{2}$ is observed, visible in particular in the interference region between $\rho^{\prime}$ and $\rho^{\prime \prime}$. The electromagnetic pion form factor obtained from the present analysis is lower (higher) than that obtained from $e^{+} e^{-} \rightarrow$ $\pi^{+} \pi^{-}$for $M_{\pi \pi}<0.8 \mathrm{GeV}\left(0.8<M_{\pi \pi}<1.8 \mathrm{GeV}\right)$. They are equal within errors for $M_{\pi \pi}>1.8 \mathrm{GeV}$.

The $Q^{2}$ dependence of the cross-section ratios $R_{\rho^{\prime}}=$ $\sigma\left(\rho^{\prime} \rightarrow \pi \pi\right) / \sigma(\rho)$ and $R_{\rho^{\prime \prime}}=\sigma\left(\rho^{\prime \prime} \rightarrow \pi \pi\right) / \sigma(\rho)$, has been studied. The ratio $R_{\rho^{\prime}}$ rises strongly with $Q^{2}$, as expected in QCD-inspired models in which the wave-function 
Table 3 The $Q^{2}$ dependence of the ratio $R_{V}$ for $V=\rho^{\prime}$ and $\rho^{\prime \prime}$. The first uncertainty is statistical, the second systematic

\begin{tabular}{llll}
\hline$Q^{2}\left(\mathrm{GeV}^{2}\right)$ & $2-5$ & $5-10$ & $10-80$ \\
\hline$R_{\rho^{\prime}}$ & $0.063 \pm 0.006 \pm 0.004$ & $0.081 \pm 0.007_{-0.005}^{+0.006}$ & $0.122 \pm 0.008_{-0.006}^{+0.005}$ \\
$R_{\rho^{\prime \prime}}$ & $0.027 \pm 0.006_{-0.003}^{+0.004}$ & $0.026 \pm 0.006 \pm 0.003$ & $0.039 \pm 0.010_{-0.005}^{+0.003}$ \\
\hline
\end{tabular}

of the vector meson is calculated within the constituent quark model, which allows for nodes in the wave-function to be present.

Acknowledgements It is a pleasure to thank the DESY Directorate for their strong support and encouragement. The remarkable achievements of the HERA machine group were essential for the successful completion of this work and are greatly appreciated. The design, construction and installation of the ZEUS detector has been made possible by the efforts of many people who are not listed as authors.

Open Access This article is distributed under the terms of the Creative Commons Attribution Noncommercial License which permits any noncommercial use, distribution, and reproduction in any medium, provided the original author(s) and source are credited.

\section{References}

1. F.D. Aaron et al. (H1 Collaboration), J. High Energy Phys. 05, 032 (2010)

2. S. Chekanov et al. (ZEUS Collaboration), PMC Phys. A 1, 6 (2007)

3. J. Breitweg et al. (ZEUS Collaboration), Phys. Lett. B 487, 273 (2000)

4. M. Derrick et al. (ZEUS Collaboration), Z. Phys. C 73, 73 (1996)

5. S. Chekanov et al. (ZEUS Collaboration), Nucl. Phys. B 718, 3 (2005)

6. M. Derrick et al. (ZEUS Collaboration), Phys. Lett. B 377, 259 (1996)

7. A. Aktas et al. (H1 Collaboration), Eur. Phys. J. C 46, 585 (2006)

8. S. Chekanov et al. (ZEUS Collaboration), Nucl. Phys. B 695, 3 (2004)

9. S. Chekanov et al. (ZEUS Collaboration), Eur. Phys. J. C 24, 345 (2002)

10. S. Chekanov et al. (ZEUS Collaboration), Phys. Lett. B 680, 4 (2009)

11. L. Frankfurt, W. Koepf, M. Strikman, Phys. Rev. D 54, 3194 (1996)

12. A.D. Martin, M. Ryskin, T. Teubner, Phys. Rev. D 56, 3007 (1997)

13. J. Nemchik, N.N. Nikolaev, B.G. Zakharov, Phys. Lett. B 339, 194 (1994)

14. J. Nemchik et al., J. Exp. Theor. Phys. 86, 1054 (1998)

15. G. Kulzinger, H.G. Dosch, H.J. Pirner, Eur. Phys. J. C 7, 73 (1999)

16. I.P. Ivanov, N.N. Nikolaev, Acta Phys. Pol. B 33, 3517 (2002)

17. F. Caporale, I.P. Ivanov, Phys. Lett. B 622, 55 (2005)

18. C. Adloff et al. (H1 Collaboration), Phys. Lett. B 541, 251 (2002)

19. L.M. Barkov et al., Nucl. Phys. B 256, 365 (1985)

20. D. Aston et al., Phys. Lett. B 92, 215 (1980)

21. A. Donnachie, H. Mirzaie, Z. Phys. C 33, 407 (1987)

22. A.B. Clegg, A. Donnachie, Z. Phys. C 62, 455 (1994)

23. I.P. Ivanov, N.N. Nikolaev, JETP Lett. 69, 294 (1999)

24. M. Atkinson et al. (Omega Photon Collaboration), Z. Phys. C 26, 499 (1985)
25. M.V. Polyakov, Nucl. Phys. B 555, 231 (1999)

26. B. Clerbaux, M.V. Polyakov, Nucl. Phys. A 79, 185 (2000)

27. J.H. Kuhn, A. Santamaria, Z. Phys. C 48, 445 (1990)

28. G. Gounaris, J.J. Sakurai, Phys. Rev. Lett. 21, 244 (1968)

29. H.B. O'Connel et al., Prog. Part. Nucl. Phys. 39, 201 (1997)

30. M. Bando et al., Phys. Rev. Lett. 54, 1215 (1985)

31. F. Caporale, I.P. Ivanov, Eur. Phys. J. C 44, 505 (2005)

32. U. Holm (ZEUS Collaboration) (ed.), The ZEUS Detector, Status Report (unpublished). DESY, 1993, available on http://www-zeus.desy.de/bluebook/bluebook.html

33. N. Harnew et al., Nucl. Instrum. Methods A 279, 290 (1989)

34. B. Foster et al., Nucl. Phys. B, Proc. Suppl. 32, 181 (1993)

35. B. Foster et al., Nucl. Instrum. Methods A 338, 254 (1994)

36. M. Derrick et al., Nucl. Instrum. Methods A 309, 77 (1991)

37. A. Andersen et al., Nucl. Instrum. Methods A 309, 101 (1991)

38. A. Caldwell et al., Nucl. Instrum. Methods A 321, 356 (1992)

39. A. Bernstein et al., Nucl. Instrum. Methods A 336, 33 (1993)

40. A. Bamberger et al., Nucl. Instrum. Methods A 382, 419 (1996)

41. A. Dwuraźny et al., Nucl. Instrum. Methods A 277, 176 (1989)

42. J. Andruszkow et al., Preprint DESY-92-066. DESY, 1992

43. M. Derrick et al. (ZEUS Collaboration), Z. Phys. C 63, 391 (1994)

44. J. Andruszkow et al., Acta Phys. Pol. B 32, 2025 (2001)

45. K. Schilling, G. Wolf, Nucl. Phys. B 61, 381 (1973)

46. M. Derrick et al. (ZEUS Collaboration), Phys. Lett. B 356, 601 (1995)

47. K. Muchorowski, PhD thesis, Warsaw University (unpublished), 1998

48. A. Kwiatkowski et al., in Proceedings of the Workshop on Physics at HERA, vol. III, ed. by W. Buchmueller, G. Ingelman (DESY, Hamburg, 1991), p. 1294

49. R. Brun et al., Geant3, Technical Report CERN-DD/EE/84-1, CERN, 1987

50. F. James et al., MINUIT, CERN DD/D506, 1994

51. K. Nakamura, et al. (Particle Data Group), J. Phys. G 37, 075021 (2010)

52. J. Breitweg et al. (ZEUS Collaboration), Eur. Phys. J. C 12, 393 (2000)

53. D. Bisello et al. (DM2 Collaboration), Phys. Lett. B 220, 312 (1989)

54. R.R. Akhmetshin et al. (CMD2 Collaboration), Phys. Lett. B 527, 161 (2002)

55. R.R. Akhmetshin et al. (CMD2 Collaboration), Phys. Lett. B 648, 26 (2007)

56. V.M. Aul'chenko et al. (CMD2 Collaboration), JETP Lett. 82, 743 (2005)

57. M.N. Achasov et al. (SND Collaboration), J. Exp. Theor. Phys. 101, 1053 (2005)

58. B. Aubert et al. (BABAR Collaboration), Phys. Rev. Lett. 103, 231801 (2009)

59. R. Barate et al. (ALEPH Collaboration), Z. Phys. C 76, 15 (1997)

60. S. Anderson et al. (CLEO Collaboration), Phys. Rev. D 61, 112002 (2000)

61. H. Hayashii, M. Fujikawa (for the Belle Collaboration), Nucl. Phys. B, Proc. Suppl. 198, 157 (2010)

62. H. Abramowicz, L. Frankfurt, M. Strikman, Surv. High Energy Phys. 11, 51 (1997)

63. I.P. Ivanov, PhD Thesis, Bonn University, hep-ph/0303035, 2003 